\title{
Elastoplastic Deformation of Silk Micro and Nanostructures
}

By Guoyou Huang ${ }^{1,2,3}$, Limei Tian ${ }^{1}$, Keng-Ku Liu ${ }^{1}$, Bo Hu${ }^{1}$, Feng $\mathrm{Xu}^{2,3}$, Tian Jian $\mathrm{Lu}^{2,3}$, Rajesh R. Naik ${ }^{4}$, Srikanth Singamaneni ${ }^{1 *}$

${ }^{1}$ Department of Mechanical Engineering and Materials Science and Institute of Materials Science and Engineering, Washington University in St. Louis, MO 63130, USA ${ }^{2}$ MOE Key Laboratory of Biomedical Information Engineering, School of Life Science and Technology, Xi'an Jiaotong University, Xi'an 710049, P.R. China;

${ }^{3}$ Bioinspired Engineering and Biomechanics Center (BEBC), Xi'an Jiaotong University, Xi'an 710049, P.R. China.

${ }^{4}$ Soft Matter Materials Branch, Materials and Manufacturing Directorate, and 711 Human Performance Wing, Wright-Patterson, Air Force Base, Dayton, $\mathrm{OH}$ 

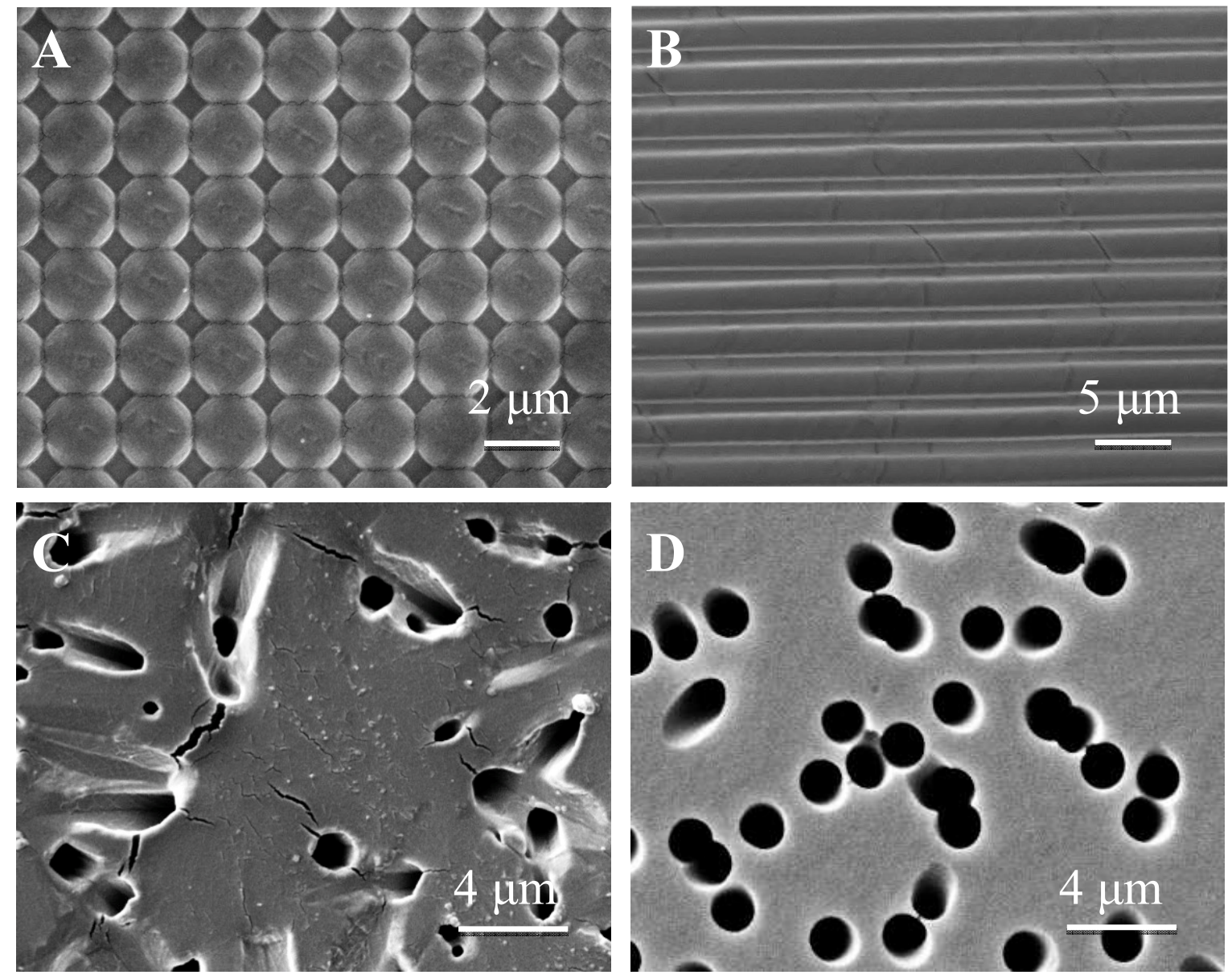

Supplementary Figure 1. Representative SEM images of PDMS templates for fabricating (A) grid patterned silk film, (B) stripe patterned silk film, (C) free-standing silk micropillars and PCTE membrane for fabricating $(F)$ free-standing hollow silk nanotubes. 

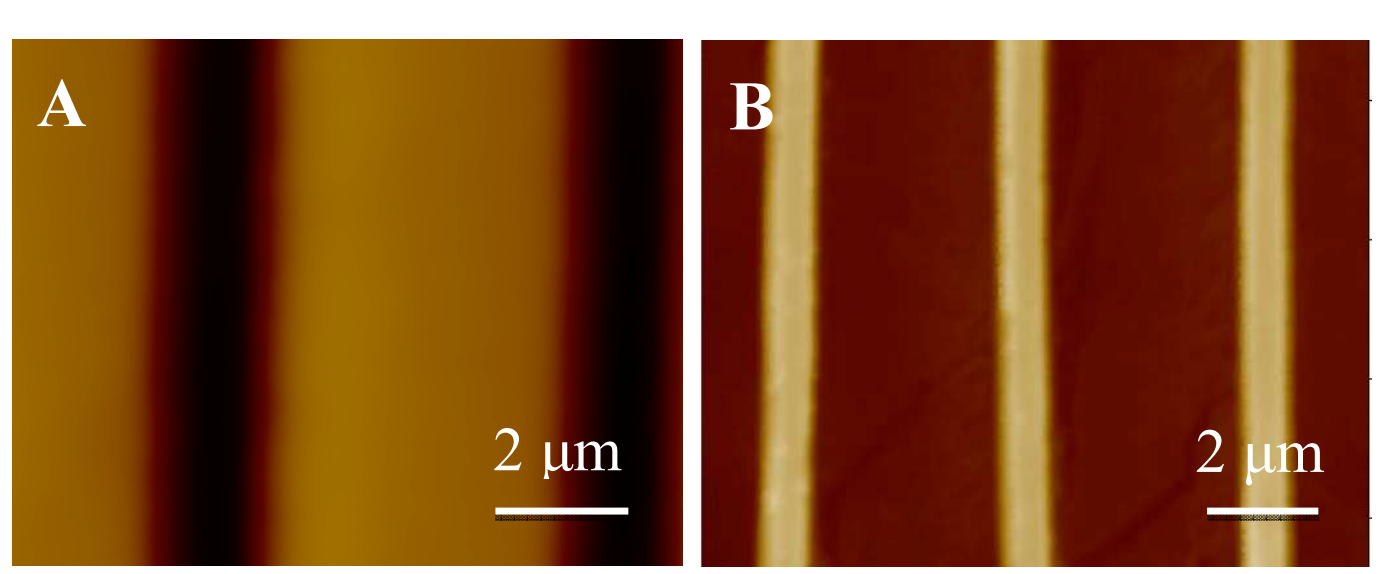

$1000 \mathrm{~nm}$
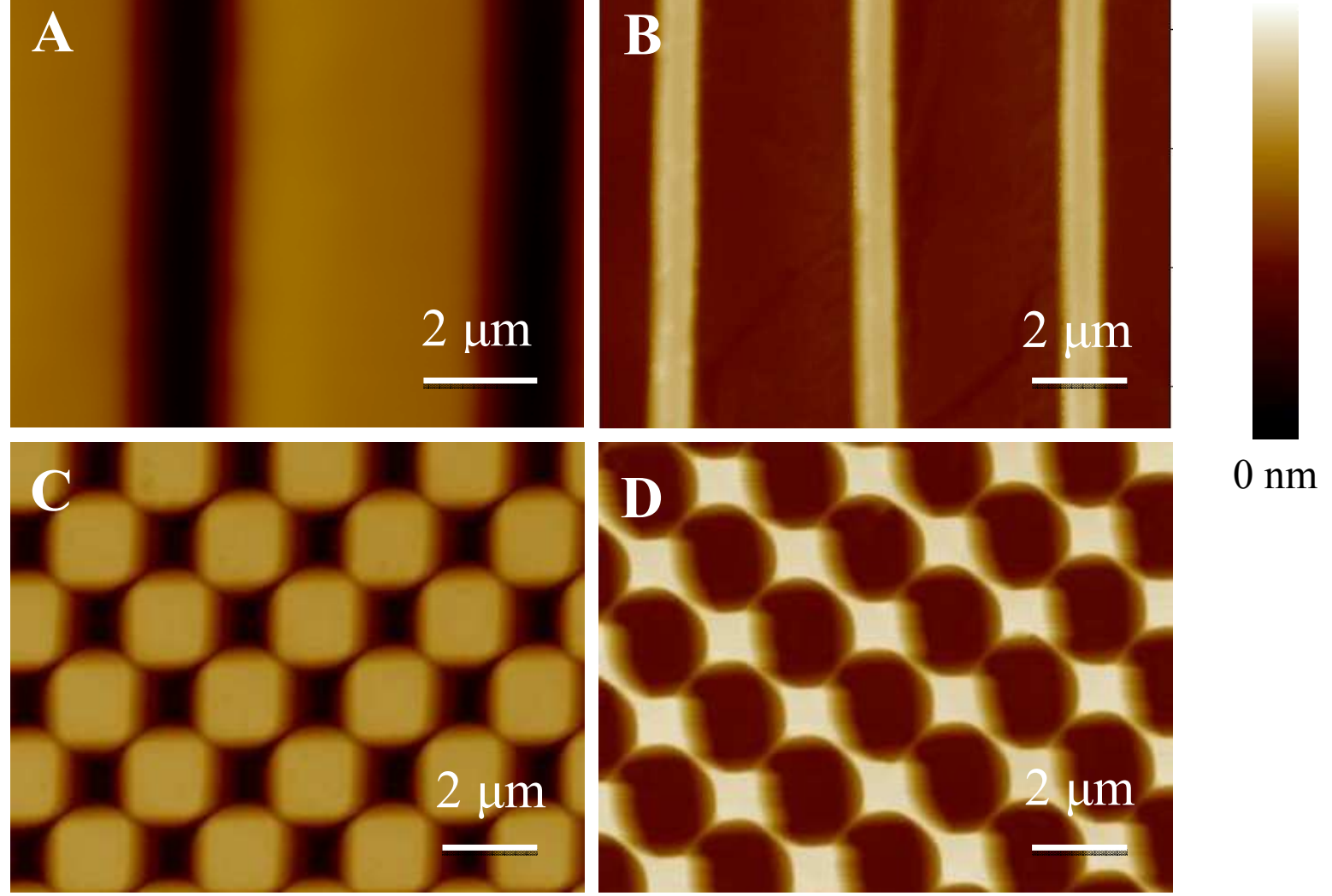

Supplementary Figure 2. AFM topography images of (A) PDMS templates for fabricating stripe patterned silk film, (B) stripe patterned silk film, (C) PDMS templates for fabricating grid patterned silk film, and (D) grid patterned silk film. 

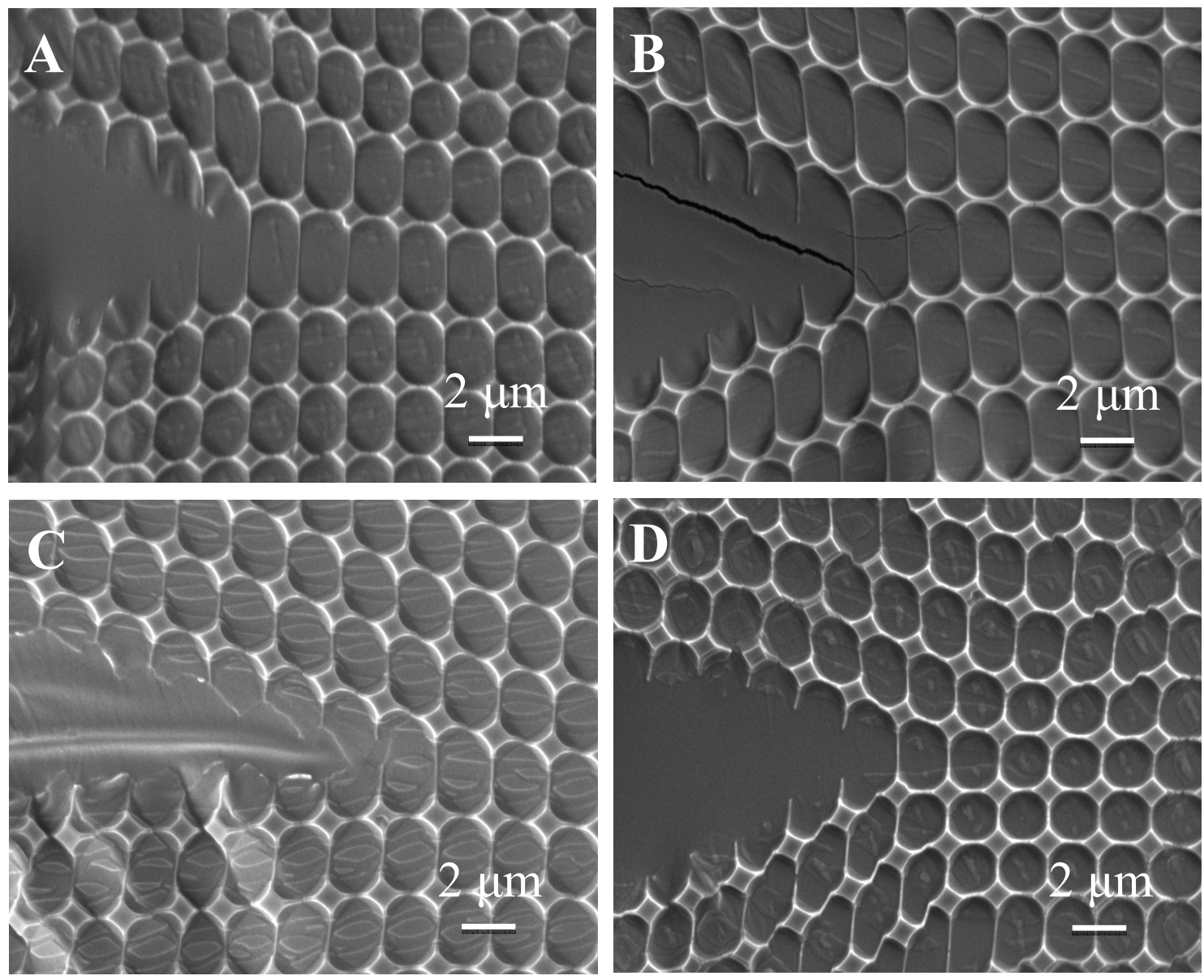

Supplementary Figure 3. SEM images of stretched grid patterned silk films treated with methanol vapor for (A) $30 \mathrm{~min},(B) 75 \mathrm{~min}$, (C) $1080 \mathrm{~min}$, and (D) methanol solution for 30 $\min$. 

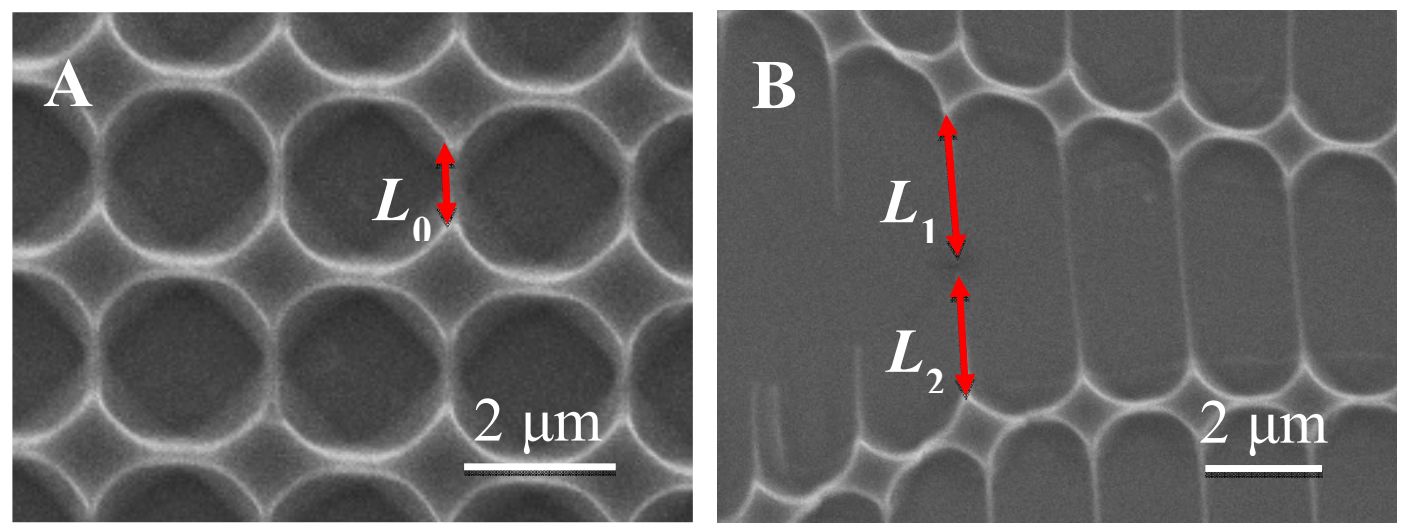

Supplementary Figure 4. (A) SEM image of grid patterned silk film on PDMS substrate. (B) SEM image of deformed grid patterned silk film on PDMS substrate. $L_{0}, L_{1}$ and $L_{2}$ indicate the length of the corresponding borders. 
A

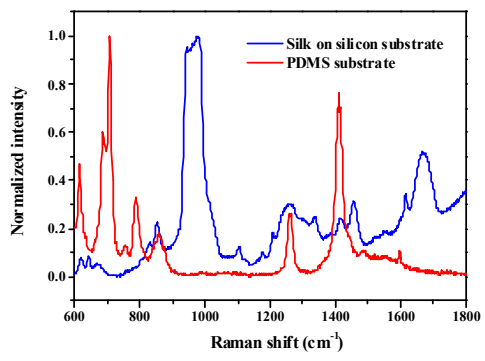

B

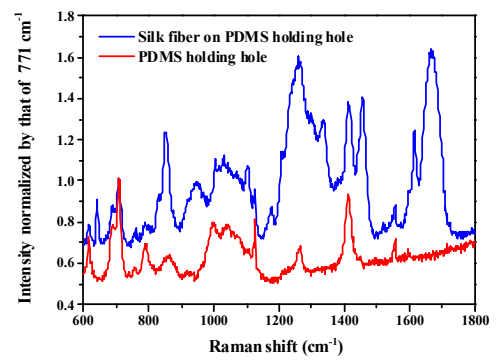

C

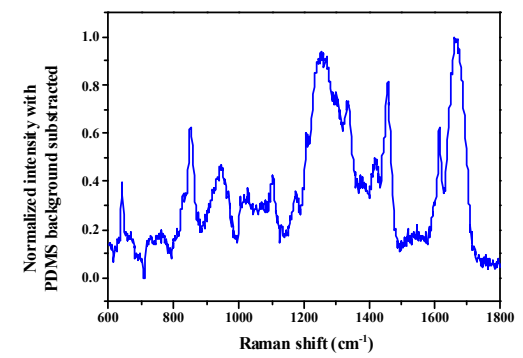

Supplementary Figure 5. (A) Raman spectra of silk on silicon substrate and that of PDMS substrate. (B) Raman spectra of PDMS well and silk patterns suspended on PDMS well, which have been normalized based on the intensity at $771 \mathrm{~cm}^{-1}$. (C) Raman spectra of silk patterns suspended on PDMS well after subtracting the PDMS background. 
Supplementary Table 1. Dimensions of the fabricated silk micro- and nanostructures

\begin{tabular}{|c|c|}
\hline Stripes & $\begin{array}{l}\text { Width: } 0.76 \pm 0.02 \mu \mathrm{m} \\
\text { Height: } 0.35 \pm 0.03 \mu \mathrm{m}\end{array}$ \\
\hline Grids & Diameter: $1.89 \pm 0.03 \mu \mathrm{m}$ \\
\hline Micropillars & $\begin{array}{l}\text { Top diameter: } 1.35 \pm 0.13 \mu \mathrm{m} \\
\text { Bottom diameter: } 1.04 \pm 0.10 \mu \mathrm{m} \\
\text { Height: } 6.90 \pm 0.42 \mu \mathrm{m}\end{array}$ \\
\hline Nanotubes & $\begin{array}{l}\text { Outer diameter: } 0.84 \pm 0.03 \mu \mathrm{m} \\
\text { Inner diameter: } 0.45 \pm 0.02 \mu \mathrm{m} \\
\text { Height: } 5.58 \pm 0.19 \mu \mathrm{m}\end{array}$ \\
\hline
\end{tabular}


Supplementary Table 2. Band assignments for regenerated B. mori silk ${ }^{[1]}$

\begin{tabular}{|c|c|c|}
\hline $\begin{array}{c}\text { Wavenumber } \\
\left(\mathrm{cm}^{-1}\right)\end{array}$ & Assignment & Refs. \\
\hline 1665 & Amide I, C=O stretching in $\beta$-sheet & {$[2-4]$} \\
\hline 1615 & Aromatic ring stretching in phenylalanine and tyrosine & {$[5]$} \\
\hline 1456 & $\begin{array}{c}\text { Amide II, } \mathrm{CH}_{3} \text { anti-symmetric bending in poly(alanine), } \mathrm{CH}_{2} \\
\text { bending in poly(alanylglycine) }\end{array}$ & {$[4,6]$} \\
\hline 1333 & $\mathrm{CH}_{3}$ symmetric bending, $\mathrm{H}^{\alpha}$ bending & {$[4]$} \\
\hline 1267 & $\begin{array}{c}\text { Amide III: } \mathrm{N}-\mathrm{H} \text { in-plane bending, } \mathrm{C}=\mathrm{O} \text { in-plane bending, } \mathrm{C}^{\alpha} \text {-C } \\
\text { stretching }\end{array}$ & {$[4,6]$} \\
\hline 1242 & Amide III, $\mathrm{B}_{2}$ symmetry: $\mathrm{H}^{\alpha} \mathrm{b}, \mathrm{N}-\mathrm{H}$ in-plane bending, $\mathrm{C}-\mathrm{N}$ & {$[4,6,7]$} \\
\hline 1210 & stretching & {$[5]$} \\
\hline 1103 & Aromatic C-H in-plane bending & {$[4,8]$} \\
\hline 1017 & Aromatic ring breathing in phenylalanine and tyrosine & {$[5]$} \\
\hline 852,830 & Fermi resonance of the tyrosine doublet & {$[9-11]$} \\
\hline
\end{tabular}

[1] M. E. Rousseau, T. Lefevre, L. Beaulieu, T. Asakura, M. Pezolet, Biomacromolecules 2004, 5, 2247.

[2] S. Krimm, Biopolymers 1983, 22, 217.

[3] W. H. Moore, S. Krimm, Biopolymers 1976, 15, 2439.

[4] W. H. Moore, S. Krimm, Biopolymers 1976, 15, 2465.

[5] A. T. Tu, Raman spectroscopy in biology : principles and applications, Vol. Chapter 3, Wiley, 1982.

[6] S. Krimm, J. Bandekar, in Adv Protein Chem, Vol. Volume 38 (Eds: J. T. E. C.B. Anfinsen, M. R. Frederic), Academic Press, 1986, 181.

[7] B. G. Frushour, P. C. Painter, J. L. Koenig, Journal of Macromolecular Science, Part C 1976, 15, 29.

[8] P. Monti, P. Taddei, G. Freddi, T. Asakura, M. Tsukada, J Raman Spectrosc 2001, 32, 103.

[9] P. Monti, G. Freddi, A. Bertoluzza, N. Kasai, M. Tsukada, J Raman Spectrosc 1998, 29, 297.

[10] M. N. Siamwiza, R. C. Lord, M. C. Chen, T. Takamatsu, I. Harada, H. Matsuura, T. Shimanouchi, Biochemistry 1975, 14, 4870.

[11] N.-T. Yu, B. H. Jo, J Am Chem Soc 1973, 95, 5033. 